\title{
TIME FOR A CULTURE CHANGE-MOVING ACADEMIA FROM DESTRUCTIVE TO CONSTRUCTIVE FEEDBACK
}

I am honored to serve as the new editor-in-chief for the Journal of Women and Minorities in Science and Engineering (JWM). Under the guidance of outgoing editorin-chief, Kimberly Douglas-Mankin, researchers have published groundbreaking work in JWM. As a field, we have taken some amazing steps forward, and now, more than ever, I am committed to a strong publication venue that supports your research about the inclusion of underrepresented and minoritized populations in science and engineering fields.

My vision as the new editor-in-chief of JWM is to create a culture of constructive feedback for the journal and for academic publishing as a whole. I envision JWM to be a journal where

- Authors feel supported in their life's work and can expect to receive fair, constructive, and timely feedback.

- Reviewers practice the art of constructive feedback in a way that positions each review to be a tangible contribution toward a constructive and supportive culture for academic publishing in STEM education.

- Associate editors create a community dedicated to holding our field to a higher standard in how we treat each other and our life's work. As individuals and as a group, JWM's associate editors will lead by example in creating a constructive culture for academic publishing.

The goal of peer review is to strengthen the quality of work in our field. Anyone who has received feedback on a manuscript - from any journal or conference - is likely to have experienced unconstructive and even destructive peer review. The problem with destructive feedback is that it does not move the field forward. Destructive feedback is an all too common, toxic element of academic culture, and it is counterproductive. It interferes with faculty and graduate student well-being. Destructive peer review can send even the best, most experienced researchers into a spiral of selfdoubt. Authors cannot develop good revisions and resubmissions in a timely manner if they are dealing with toxic reviews. If we want to move the field forward, we need to change the type of feedback we provide to authors. What would happen if we each committed to offer the kind of high-quality, practical guidance we all desire?

I want us to challenge ourselves to support each other by holding ourselves to a higher standard of peer review. Constructive feedback is a type of mentoring for the research community. Rather than being gatekeepers for each other's work, we foster each other's ability to perform at our highest level as peer mentors, which moves the field forward. Constructive mentoring through peer review increases the research capacity of individual researchers because it helps authors be more productive. It 
helps authors understand the strengths of their research and the most effective ways to frame it. Constructive peer review increases the impact of our field because it leads to stronger publications. And, constructive feedback assists editors in making decisions because it helps us/them understand the potential of a manuscript, what to prioritize, how to maximize revision requests, and ultimately, how to make informed decisions more quickly.

Constructive peer review is a form of mentoring because it expands our individual capacity for excellence, increases the quality of our collective work, and helps us maintain our well-being. How can you contribute to a culture of mentoring with constructive feedback with your next review? Consider these elements of constructive feedback: Constructive feedback

- Is specific. Constructive feedback uses examples from the manuscript where possible to help the author and editor understand what you mean.

- Is actionable. Criticisms of the manuscript are followed by suggestions for improvement.

- Is prioritized. It is easy for an author (or editor) to get overwhelmed by the volume of feedback in a review. Prioritized feedback labels major and minor concerns. It is organized in a way that allows the reader to easily understand which points to prioritize in a revision, either according to sections of the paper or thematically. Prioritized feedback makes clear what concerns are global (across the article as a whole) and which concerns are local to a specific section (such as in the research design).

- Is balanced. Constructive feedback describes the document's strengths as well as areas for improvement. It is important for the reviewer to demonstrate enthusiasm to editor through the language chosen, since it tells the editor which elements should continue through a revision.

- Acknowledges the reviewer's positionality. Not only does understanding that a reviewer is someone who does $\mathrm{X}$ or knows about $\mathrm{Y}$ demonstrate that the reviewer knows what they are talking about, but also, understanding a reviewer's positionality helps the author and editor place the reviewer's comments in context. In situations where mixed reviews are received, it helps the editor prioritize and make decisions about which critiques require a response from the author.

- Contains positive, tactful, and nonthreatening language that addresses the document, not the author. By focusing on the manuscript and not the author, constructive feedback avoids personal criticisms.

- Is speedy. We all need feedback. Our careers are dependent on it. The great work we are doing cannot be shared and used by others if it is not published in a timely manner.

Destructive peer review is academic bullying at its worst. It should be our community's goal to uplift each other and acknowledge the bravery of presenting our life's 
work to the world while offering ways to continuously improve. Will you join me in creating a constructive culture for academic publishing?

I would love to hear from you about your ideas for mentoring through constructive peer review.

Julie P. Martin

Editor-in-Chief 
